\title{
ORIGINAL
}

\section{DISTRIBUCIÓN EN ESPAÑA DEL CUIDADO FORMAL E INFORMAL A LAS PERSONAS DE 65 Y MÁS AÑOS EN SITUACIÓN DE DEPENDENCIA (*)}

\section{Jesús Rogero-García}

Instituto de Economía, Geografía y Demografía (IEGD). Centro de Ciencias Humanas y Sociales (CCHS). Consejo Superior de Investigaciones Científicas (CSIC).

\section{RESUMEN}

Fundamento: La distribución entre cuidado formal e informal a personas mayores dependientes es una cuestión relativamente inexplorada en España. El objetivo de este trabajo es analizar cómo se distribuye el cuidado informal y formal entre las personas mayores dependientes en España, así como las condiciones sociales y económicas en que se desarrollan estos tipos de cuidado.

Métodos: Se utilizó una muestra de personas mayores de 64 años dependientes procedente de la Encuesta Nacional de Salud 2003. Se realizaron tablas de contingencia para conocer la distribución del cuidado entre las personas mayores españolas, y se construyeron varios modelos de regresión logística binaria para identificar los factores asociados a la recepción de los diferentes tipos de cuidado.

Resultados: El 7,5\% de las personas mayores que necesitan cuidado no lo reciben. De quiénes lo reciben, el $89,4 \%$ recibe cuidado informal, el $14,8 \%$ servicios privados y el $8,1 \%$ servicios públicos. Cuidado formal e informal se combinan en el $11,9 \%$ de los casos. Quienes viven en hogares con ingresos superiores a $900 €$ tienen 5 veces más probabilidades de recibir servicios privados en lugar de cuidado informal que los hogares de menos de $600 €$, y los mayores de 84 años tienen 6 veces más probabilidades de recibir apoyo público que los que tienen entre 65 y 74 .

Conclusiones: Los hombres tienen el doble de probabilidades de recibir sólo cuidado informal, y las mujeres tienen 3 veces más probabilidades de recibir cuidado formal e informal combinado en lugar de un tipo de cuidado. El cuidado formal sirve de complemento al informal, y los servicios de cuidado privados tienen mayor presencia que los públicos. Ser varón, vivir en hogares de mayor tamaño y con menos recursos socioeconómicos incrementa la probabilidad de recibir cuidado informal.

Palabras clave: Anciano. Cuidadores. Servicios de atención de salud a domicilio. Atención domiciliaria de salud. España.

Correspondencia:

C/ Albasanz, 26-28

Despacho 3E10

28037 Madrid

jesus.rogero@cchs.csic.es

\section{ABSTRACT \\ Distribution of Formal and Informal Home Care for People Older than 64 years in Spain 2003}

Background: There has been a relative lack of research examing the distribution of care to elderly dependent people in Spain. The aim of this paper is to analyse how formal and informal care is provided to elderly dependent people in Spain and to assess the socio-economic conditions in which the different kinds of care emerge.

Methods: This study is based on a sample of the elderly dependent population selected from those who reported the need of care in the 2003 Spanish Health Survey. The distribution of care among older people was cross-tabulated to identify the type of care, while regression models were used to identify the socio-economic characteristics of people receiving formal and/or informal care.

Results: Around $7.5 \%$ of the elderly people who need care do not receive it. Among those who do receive it, $89.4 \%$ receive informal care, $14.8 \%$ private care and $8.1 \%$ public care. $11.9 \%$ of elderly people in the study receive a mix of formal an informal care. Elderly people living in households in which the monthly income exceeds EUR900 are five times more likely than people living in households in which the monthly income is less than EUR 600 to receive private care instead of public care. People older than 84 years are six times more likely to receive public care than people aged between 65 and 74 .

Conclusions: Men are two times more likely than women to receive exclusively informal care. Women are three times more likely than men to receive both kinds of care simultaneously. Formal care complements informal care. Private care is more common than public care. Men, people living in larger-sized households, and people with fewer socioeconomic resources are more likely to receive informal care.

Key words: Aged. Caregivers. Home care services. Home nursing. Spain.

(*) Trabajo desarrollado durante el disfrute de la beca predoctoral I3P-BPD2003-1 (proyecto de referencia SEC200200504) del Consejo Superior de Investigaciones Científicas. 


\section{INTRODUCCIÓN}

La actividad de cuidar se ha realizado y se realiza desde diferentes instituciones sociales: el Estado (residencias, hospitales, etc.), la familia (cuidado de abuelas a nietos, de hijos a padres, etc.), el mercado (servicios con fines de lucro) $u$ otras instituciones (organizaciones sin fines de lucro, etc.). El tipo de cuidado viene dado por la institución o personas que lo proveen, por la situación de quien lo recibe, y por el contexto en el que se desarrolla. Las personas requieren cuidado cuando necesitan ayuda para desarrollar alguna de las actividades de la vida diaria (AVD), que son aquellas que una persona ha de realizar diariamente para poder vivir de forma autónoma, integrada en su entorno habitual y cumpliendo su rol social. Suelen diferenciarse en actividades básicas (autocuidado, movilidad en el hogar y comunicación) e instrumentales (tareas domésticas, administración del hogar y movilidad en el entorno) ${ }^{1}$. Cuando una persona tiene dificultades para desarrollar las actividades de la vida diaria, se presentan cuatro posibilidades: (1) que reciba cuidado informal (2) que reciba cuidado formal, (3) que reciba cuidado formal e informal o (4) que no reciba ningún tipo de cuidado.

El concepto de cuidado informal ha sido utilizado para aludir a un tipo de apoyo que se caracteriza porque (a) es desarrollado por personas de la red social del receptor del cuidado y (b) se provee de forma voluntaria, sin que medie ninguna organización ni remuneración ${ }^{2}$. El cuidado informal suele distinguir tres categorías de ayuda: apoyo material o instrumental, apoyo informativo o estratégico y apoyo emocional ${ }^{3,4}$. Una parte de la atención provista a los mayores por sus cuidadores informales se traduce también en tareas propias de los profesionales de la salud, como el diagnóstico de enfermedades o la provisión de medicamentos ${ }^{5}$.

El cuidado formal se ha definido como aquellas acciones que un profesional oferta de forma especializada, y que va más allá de las capacidades que las personas poseen para cuidar de sí mismas o de los demás ${ }^{6}$. Existen dos tipos básicos de cuidado formal: el que se provee desde las instituciones públicas, y el que se contrata a través de las familias. Los servicios públicos de cuidado pasan a través de una organización burocratizada y están, al menos en parte, financiados públicamente. Por otro lado, las familias recurren cada vez más a servicios privados de cuidado para hacer frente a la atención de sus miembros dependientes en España, debido sobre todo a la disponibilidad de mano de obra barata procedente de la inmigración.

Se ha estimado que hasta el $88 \%$ del cuidado de la salud en España se realiza no remuneradamente ${ }^{7}$. ¿Qué factores actúan para que se desarrolle una combinación u otra de cuidado formal e informal en el hogar? Según una investigación de Chappell y Blandford realizada sobre población canadiense $^{8}$, el cuidado formal aparece cuando la severidad de la discapacidad genera necesidades que exceden las posibilidades de atención de los cuidadores informales. Phillips ${ }^{9}$, por su parte, realizó un estudio con datos de 1991 y 1992 sobre población adulta (entre 16 y 98 años) de rentas bajas en Estados Unidos, en el que controló el tipo de problema que causa la discapacidad, y no halló diferencias significativas en la recepción de cuidado formal según edad, nivel de dependencia y duración de la discapacidad.

De acuerdo con el estudio de Kemper en Estados Unidos (datos de 1988), la existencia de una red de apoyo es también determinante en el tipo de cuidado recibido por los mayores ${ }^{10}$. En esta línea, Herlitz ${ }^{11}$ estudió la distribución de cuidado formal e informal en el hogar de los mayores en dos distritos urbanos y dos distritos rurales del centro y sur de Suecia, y halló que las personas que vivían solas tenían menos probabilidades de recibir cuidado informal que el resto. La 
composición y el tamaño de la red social de los mayores está relacionada con la edad: a medida que ésta se incrementa, el número de potenciales cuidadores de la red social se reduce.

Los hombres tienen más probabilidades de recibir cuidado informal que las mujeres, y las mujeres tienen más probabilidades de recibir cuidado formal o ambos tipos de cuidado conjuntamente. Estas diferencias tienen su origen en que las mujeres viven más que los hombres y con más enfermedades crónicas discapacitantes ${ }^{12}$, y en que los roles sociales y las normas culturales de género motivan una mayor disposición de las mujeres hacia el cuidado de los hombres que a la inversa ${ }^{13}$. La situación socioeconómica es también relevante en el cuidado recibido. El citado estudio de Kemper halló que el uso de servicios de cuidado formal se incrementa y el cuidado informal se reduce a medida que aumentan los ingresos.

Existe una influencia mutua entre cuidado formal e informal: los cuidadores informales juegan un importante papel en la elección y provisión del cuidado formal, y la disponibilidad y el desarrollo de los servicios formales influye decisivamente en la intensidad y el tipo de cuidado informal ${ }^{14}$. Dicho de otro modo, los servicios formales interactúan dinámicamente con el sector informal $^{15}$. La naturaleza de la relación entre cuidado formal e informal continúa siendo uno de los principales desafíos para los investigadores en este campo ${ }^{16}$. Desde nuestro punto de vista, esta relación bascula en torno a dos conceptos básicos: (1) la complementariedad, es decir, "la cualidad o circunstancia que se añade a otra para hacerla íntegra o perfecta"; y (2) la suplementariedad o "reemplazo de algo por otra cosa" 17. Los modelos teóricos sobre la relación entre cuidado formal e informal pueden sintetizarse en tres:

El modelo de cuidado suplementario postula que los cuidadores familiares pro- veen la mayoría del cuidado, y que la utilización del cuidado formal sirve para sustituir temporal o circunstancialmente el cuidado informal ${ }^{18}$.

El modelo compensador jerárquico sugiere que los mayores prefieren ser cuidados en primer lugar por su cónyuge, luego por sus hijos, otros miembros de la familia y, por último, por cuidadores formales. Este modelo es compatible con el modelo suplementario, pues asume que el cuidado provisto por cualquier cuidador se puede sustituir por otro, aunque en el orden mencionado. Paralelamente, tanto familiares como no familiares pueden participar en el cuidado, pero normalmente realizando actividades diferentes ${ }^{19}$.

\section{El modelo de cuidado complementario o} de especificidad de tareas plantea que el cuidado formal aparece cuando las tareas demandadas exceden las capacidades de los cuidadores informales. Los cuidadores informales y los proveedores formales de cuidado proporcionan diferentes tipos de cuidado en función de sus características ${ }^{20}$.

Los tres modelos coexisten y tienen diferente protagonismo en función del contexto social, económico y político. Algunos servicios de respiro (atención formal temporal que tiene entre sus objetivos proporcionar a los cuidadores descanso y/o tiempo libre) o los centros de día para mayores, pueden ser un ejemplo de suplementariedad entre cuidado formal e informal, cuya eficacia en la reducción de la carga subjetiva de cuidado ha sido probada en países como Holanda ${ }^{21}$. Los servicios de telemedicina, a través de los cuales se provee atención médica o de enfermería en momentos puntuales, son un ejemplo de cómo los servicios formales pueden complementar la acción de los cuidadores informales.

El presente artículo aborda la distribución del cuidado formal e informal entre las personas mayores dependientes en España. 
Sus objetivos específicos son (1) conocer la distribución y composición del cuidado entre los mayores españoles dependientes según agente proveedor, (2) examinar el modelo de relación entre el cuidado formal e informal en España, e (3) identificar los factores sociodemográficos asociados con la recepción de cuidado formal e informal.

\section{MATERIAL Y MÉTODOS}

La fuente de datos para realizar el análisis fue la Encuesta Nacional de Salud 2003 (ENSE 2003), llevada a cabo por el Ministerio de Sanidad y Consumo y el Instituto Nacional de Estadística. La ENSE 2003 es representativa a nivel nacional y contiene datos sobre 6.134 personas mayores de 64 años. El método de recogida de información de la ENSE 2003 fue la entrevista personal que se complementa mediante entrevista telefónica en casos excepcionales. Si la persona que resultó seleccionada no pudo facilitar los datos, bien por problemas de edad, enfermedad o ausencia repetida, se solicitaba la información a otra persona residente del hogar que estuviera "suficientemente informada sobre los datos que se solicitan sobre el sujeto de entrevista y capacitada para hacerlo"22. La ENSE 2003 permite analizar la población receptora de cualquier tipo de cuidado, lo que posibilita estimar el peso de los diferentes proveedores entre la población de mayores, y relacionarlos con sus características sociodemográficas.

Se seleccionó una submuestra con todos los individuos que reunían las siguientes características: (a) tener 65 ó más años, (b) no estar institucionalizados, es decir, no vivir en establecimientos colectivos como residencias u hospitales, (c) declarar tener "alguna dificultad para realizar las actividades de la vida diaria (salir de casa, vestirse, aseo personal, comer, etc.)", (d) declarar necesitar "algún tipo de ayuda o cuidado de otras personas para realizar las actividades de la vida diaria", y (e) señalar el tipo de cuidado que reciben, o bien que no reciben ningún tipo de cuidado (INE, 2003). Reunieron estos criterios un total de 1.156 individuos (327 varones y 829 mujeres).

La variable dependiente fue el tipo de cuidado según proveedor, distribuido en cuatro categorías: cuidado formal, cuidado informal, cuidado formal e informal y no recibe cuidado. El cuidado informal es aquel provisto por familiares, amigos o vecinos, independientemente de que reciban o no algún dinero a cambio (sólo el 1,7\% de los cuidadores informales recibió algún dinero a cambio del cuidado). En la muestra seleccionada no aparecían voluntarios que proveyeran cuidado a través de una organización, y se eliminaron dos casos en los que no se identificaba la relación con el cuidador. El cuidado formal es el suministrado por profesionales que cobran a cambio de ello. Son cuidadores formales privados tanto "las personas empleadas en el hogar dedicadas principalmente al cuidado de las personas" como "las personas empleadas en el hogar dedicadas principalmente a otras tareas" $^{23}$, ya que se considera que ambas actividades constituyen una ayuda para el desarrollo de las Actividades de la Vida Diaria. Las variables independientes se diferenciaron en tres tipos. En primer lugar, las características del receptor: sexo, edad (6574, 75-84 u 85 ó más años), nivel de dificultad (moderada o severa), tipo de problema que da lugar a la dificultad (físico, o mental o físico y mental), nivel de estudios ( $\sin$ estudios, primarios o secundarios, o postsecundarios) y estado civil (casado/en pareja, soltero u otra situación -viudo, separado o divorciado-). En segundo lugar, las características de su hogar: número de miembros (una, dos, tres o cuatro o más personas) e ingresos mensuales (hasta $600 €, 601-900$ $€$ €, 901-1.200 €, 1.201-1.800 € ó más de $1.800 €)$. Finalmente, se exploró también el efecto del tamaño del municipio de residencia (menos de 10.000, entre $10.001 \mathrm{y}$ 100.000 ó más de 100.000 habitantes). 
El análisis estadístico consistió en realizar tablas de contingencia y modelos de regresión logística binaria en pasos hacia delante. Estos modelos permiten estudiar si una variable de respuesta dicotómica está asociada estadísticamente a uno o más factores, y predicen la probabilidad de que ocurra un suceso bajo ciertas circunstan$\operatorname{cias}^{24}$. En este caso, los modelos de regresión logística exploran la probabilidad que tienen las personas mayores que necesitan ayuda de recibir diferentes tipos de cuidado, en función de las variables independientes referidas. Esa probabilidad se expresa a través de los odds ratio, que son el cociente entre la probabilidad de que un evento suceda y la probabilidad de que no suceda, en función de un cambio en la variable predictora respecto a la categoría de referencia. Por ejemplo, puede estudiarse si la probabilidad de recibir cuidado es mayor cuando se padece discapacidad mental y física que cuando la discapacidad es sólo física (categoría de referencia). Un odd ratio para la categoría "discapacidad mental y física" igual a 1 significará que existe la misma probabilidad $(0,5)$ de recibir cuidado en ambos casos. Un odd ratio igual a 4 indicará que la probabilidad de recibir cuidado es cuatro veces mayor (una probabilidad de 0,8 ) en los casos de discapacidad mental y física, en comparación con los casos de sólo discapacidad física. La $\mathrm{R}^{2}$ de Nagelkerke indica el porcentaje de varianza explicado por el modelo, y constituye un indicador adecuado para reflejar su ajuste global ${ }^{25}$. La $\mathrm{R}^{2}$ de Nagelkerke es una modificación de la $\mathrm{R}^{2}$ de Cox-Snell. Su valor máximo es 1 y su valor mínimo 0. Las variables independientes introducidas en los modelos, así como su categorización, se detallan en la tabla 1 .

\section{RESULTADOS}

El porcentaje de personas mayores no institucionalizadas que necesitaban ayuda en las Actividades de la Vida Diaria fue del $22,3 \%$, un $16,6 \%$ de los varones y un $26,6 \%$ entre las mujeres. De los mayores que necesitaban ayuda el 92,5\% recibía cuidado de algún tipo y el 7,5\% declaraba no recibirlo. Al aplicar estos porcentajes a las cifras de población del Padrón de Habitantes 2003, fue posible estimar que 1.397.111 mayores

Tabla 1

Variable dependiente y variables independientes

\begin{tabular}{|c|c|c|}
\hline \multicolumn{2}{|c|}{$\begin{array}{l}\text { Variable dependiente: } \\
\text { tipo de cuidado recibido }\end{array}$} & $\mathbf{N}$ \\
\hline \multicolumn{2}{|l|}{ Sólo cuidado informal } & 765 \\
\hline \multicolumn{2}{|l|}{ Sólo cuidado privado } & 99 \\
\hline \multicolumn{2}{|c|}{ Sólo cuidado de servicios sociales } & 66 \\
\hline \multicolumn{2}{|c|}{ Cuidado informal y privado } & 74 \\
\hline \multicolumn{2}{|c|}{ Cuidado informal y de servicios sociales } & 45 \\
\hline \multicolumn{2}{|l|}{ No recibe cuidado } & 107 \\
\hline $\begin{array}{c}\text { Variables } \\
\text { independientes: } \\
\text { características } \\
\text { de los mayores } \\
\text { y su entorno }\end{array}$ & Categorías & $\mathbf{N}$ \\
\hline \multirow[t]{3}{*}{ Edad } & $65-74$ & 362 \\
\hline & $75-84$ & 496 \\
\hline & $84+$ & 298 \\
\hline \multirow[t]{2}{*}{ Sexo } & Varón & 327 \\
\hline & Mujer & 829 \\
\hline \multirow[t]{2}{*}{ Nivel de dificultad } & Moderada & 687 \\
\hline & Severa & 469 \\
\hline \multirow[t]{2}{*}{ Tipo de problema } & Físico & 942 \\
\hline & Mental o físico y mental & 214 \\
\hline \multirow[t]{4}{*}{ Tamaño del hogar } & 1 & 396 \\
\hline & 2 & 494 \\
\hline & 3 & 138 \\
\hline & 4 ó más & 128 \\
\hline \multirow[t]{3}{*}{ Estado civil } & Casado o en pareja & 459 \\
\hline & Soltero & 103 \\
\hline & Viudo, separado o divorciado & 594 \\
\hline \multirow[t]{3}{*}{ Nivel educativo } & Sin estudios & 579 \\
\hline & Primarios o equivalentes & 474 \\
\hline & Secundarios o postsecundarios & 103 \\
\hline \multirow[t]{4}{*}{ Ingresos del hogar } & Menos de $600 €$ & 457 \\
\hline & $601-900 €$ & 244 \\
\hline & Más de $900 €$ & 246 \\
\hline & No constan ingresos & 209 \\
\hline \multirow[t]{3}{*}{ Tamaño del municipio } & Menos de $\mathbf{1 0 . 0 0 0}$ & 410 \\
\hline & $10.001-100.000$ & 372 \\
\hline & Más de 100.000 & 374 \\
\hline
\end{tabular}

(Categoría de referencia en negrita). 
no institucionalizados necesitaban cuidado (tabla 2). De ellos 1.292.893 recibían algún tipo de cuidado, y 104.218 no recibían ninguno. Entre quienes recibían cuidado, en el 89,4\% disponía de cuidado era informal (1.155.108 mayores), y para el $77,5 \%$ era su única fuente de apoyo (1.001.376 mayores). El 6,4\% recibía únicamente cuidado formal privado y el $4 \%$ exclusivamente cuidado procedente de servicios públicos. El $8,1 \%$ de las personas mayores con ayudas personales combinaba el cuidado informal con servicios de cuidado pagados por ellos mismos o sus familias (cuidado privado), y el $3,8 \%$ con servicios públicos. Un $0,3 \%$ declaró recibir cuidado formal público y privado.

El $9,1 \%$ de las personas mayores que recibía cuidado informal disponía también
Tabla 2

Estimación de la población de mayores no institucionalizados que reciben ayuda, según tipo de apoyo recibido. España 2003

\begin{tabular}{|l|r|r|}
\hline & Población & \multicolumn{1}{|c|}{$\%$} \\
\hline Total & 1.292 .893 & 100,0 \\
\hline Sólo reciben cuidado informal & 1.001 .376 & 77,5 \\
\hline Cuidado informal y cuidado formal privado & 104.787 & 8,1 \\
\hline Cuidado formal privado & 82.817 & 6,4 \\
\hline Cuidado informal y cuidado formal público & 48.945 & 3,8 \\
\hline Cuidado formal público & 51.279 & 4,0 \\
\hline Cuidado formal público y privado & 3.689 & 0,3 \\
\hline
\end{tabular}

Fuente: Elaboración propia sobre datos del Padrón municipal 2003 (servidor Web del INE: http://www.ine.es, 2008) y microdatos de la Encuesta Nacional de Salud 2003 (INE).

de servicios de cuidado privados y el 4,3\% tenía apoyo de servicios sociales. Cuanto menos cercana era la relación entre el cuidador informal y la persona dependiente mayor era la probabilidad de utilizar servi-

Figura 1

Cuidado formal recibido según relación con cuidador informal (porcentajes)

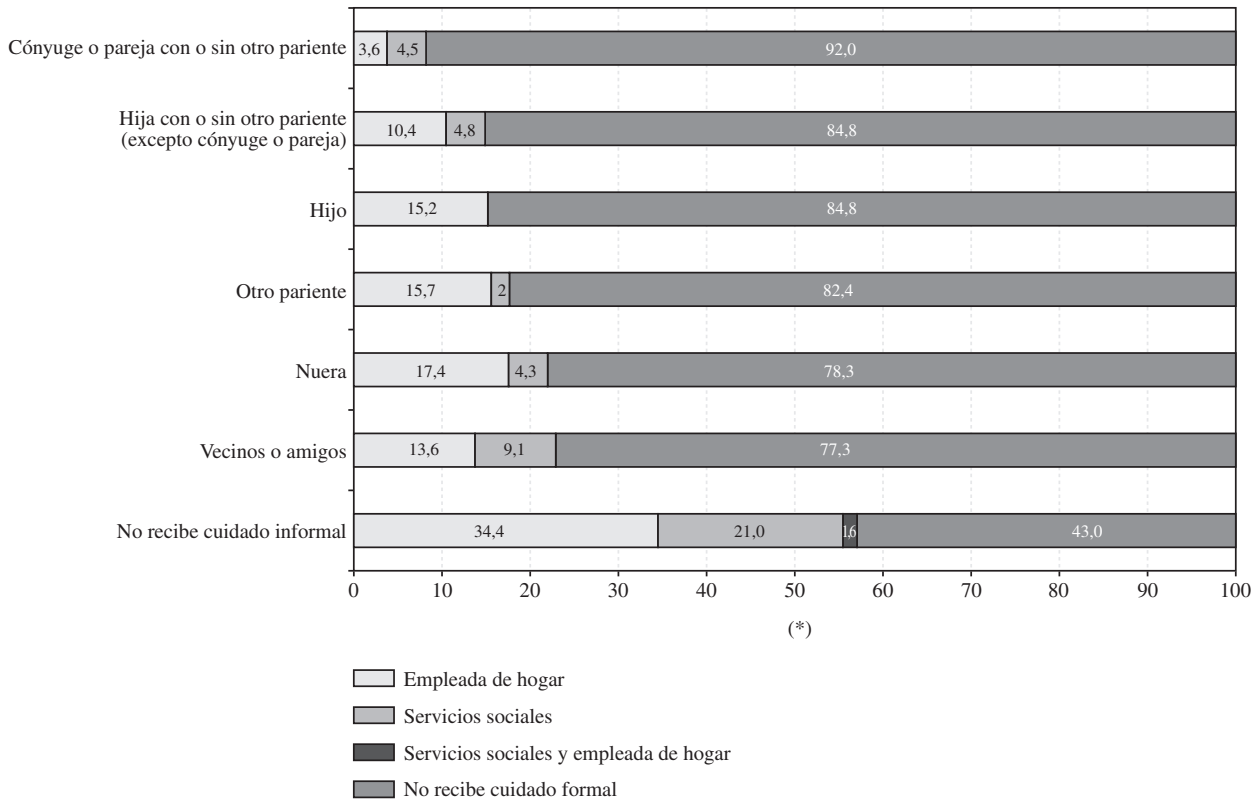

N: Total, 1156. Cónyuge o pareja con o sin otro pariente, 243; hija con sin otro pariente, 251; hijo, 52; nuera, 38; Otro pariente, 103; Vecinos o amigos, 31; cónyuge-pareja e hija, 46; cónyuge-pareja y otro pariente, 25; hija y otro pariente (excepto pareja), 95; Sin cuidado informal, 272. Fuente: Elaboración propia sobre microdatos de la Encuesta Nacional de Salud 2003 (Instituto Nacional de Estadística). 
cios formales de cuidado (figura 1). El 57\% de las personas mayores con necesidad de ayuda que no disponían de cuidado informal sí recibía otros tipos de apoyo: el $34,4 \%$ de servicio doméstico, el $21 \%$ de servicios sociales y un 1,6\% de ambos. En cambio, el cuidado formal sí se complementaba frecuentemente con cuidado informal: el $55,1 \%$ de las personas que recibían ayuda de un cuidador remunerado eran ayudados también por familiares o amigos, y el 46,8\% de los que recibían ayuda de servicios sociales tenía a su vez cuidado informal.

A continuación se describen los resultados de los modelos de regresión logística binaria que exploraron las variables asociadas a la recepción de diferentes combinaciones de cuidado. La tabla 3 muestra los

Tabla 3

Modelos de regresión logística de los factores asociados a la recepción de cuidado informal

\begin{tabular}{|c|c|c|}
\hline \multicolumn{3}{|c|}{ Modelo 1. Recibe cuidado informal (ref: no recibe ningún tipo de cuidado) } \\
\hline Variables & Sig. & Odds ratio \\
\hline $\begin{array}{l}\text { Sexo (ref: Varón) } \\
\text { Mujer }\end{array}$ & 0,025 & 0,521 \\
\hline $\begin{array}{l}\text { Tamaño del hogar (ref: } 1) \\
2 \\
3 \\
4 \text { o más }\end{array}$ & $\begin{array}{l}0,000 \\
0,000 \\
0,000 \\
0,000\end{array}$ & $\begin{array}{c}5,697 \\
9,320 \\
25,554\end{array}$ \\
\hline $\begin{array}{l}\text { Estado civil (ref: casado o en pareja) } \\
\text { Soltero } \\
\text { Viudo, separado o divorciado }\end{array}$ & $\begin{array}{l}0,004 \\
0,606 \\
0,005\end{array}$ & $\begin{array}{l}0,789 \\
3,139\end{array}$ \\
\hline $\begin{array}{l}\text { Tipo de problema (ref: físico) } \\
\text { Mental o mental y físico }\end{array}$ & 0,008 & 3,898 \\
\hline Constante & 0,296 & 1,633 \\
\hline \multicolumn{3}{|c|}{$\begin{array}{l}\text { N: 872. Porcentaje pronosticado correctamente por el modelo: } 90,588 \% \text {. } \mathrm{R}^{2} \text { de Nagelkerke: } 0,173 \text {. Modelo por pasos hacia delante. Paso } 1^{\circ} \text {. Tamaño de } \\
\text { hogar. Paso } 2^{\circ} \text {. Tipo de problema. Paso } 3^{\circ} \text {. Estado civil. Paso } 4^{\circ} \text {. Sexo. }\end{array}$} \\
\hline \multicolumn{3}{|c|}{ Modelo 2. Recibe cuidado informal o de empleados de hogar (ref: recibe cuidado informal) } \\
\hline Variables & Sig. & Odds ratio \\
\hline $\begin{array}{l}\text { Tamaño del hogar (ref: } 1 \text { ) } \\
2 \\
3 \\
4 \text { o más }\end{array}$ & $\begin{array}{l}0,000 \\
0,000 \\
0,000 \\
0,000\end{array}$ & $\begin{array}{l}0,065 \\
0,065 \\
0,029\end{array}$ \\
\hline $\begin{array}{l}\text { Nivel educativo (ref: sin estudios) } \\
\text { Estudios primarios o equivalentes } \\
\text { Estudios secundarios o superiores }\end{array}$ & $\begin{array}{l}0,002 \\
0,163 \\
0,000 \\
\end{array}$ & $\begin{array}{l}1,563 \\
4,222\end{array}$ \\
\hline $\begin{array}{l}\text { Ingresos del hogar (ref: menos de } 600 €) \\
601-900 € \\
\text { Más de } 900 € \\
\text { No constan ingresos }\end{array}$ & $\begin{array}{l}0,007 \\
0,007 \\
0,001 \\
0,013\end{array}$ & $\begin{array}{l}3,452 \\
5,246 \\
3,105\end{array}$ \\
\hline Constante & 0,000 & 0,176 \\
\hline \multicolumn{3}{|c|}{$\begin{array}{l}\text { N: 866. Porcentaje pronosticado correctamente por el modelo: } 92,773 \% \\
\mathrm{R}^{2} \text { de Nagelkerke: } 0,251 \text {. Modelo por pasos hacia delante. Paso } 1^{\circ} \text {. Tamaño del hogar. Paso } 2^{\circ} \text {. Nivel educativo. Paso } 3^{\circ} \text {. Ingresos del hogar. }\end{array}$} \\
\hline \multicolumn{3}{|c|}{ Modelo 3. Recibe cuidado informal o de servicios sociales (ref: recibe cuidado informal) } \\
\hline Variables & Sig. & Odds ratio \\
\hline $\begin{array}{l}\text { Tamaño del hogar (ref: } 1) \\
2 \\
3 \\
4 \text { o más }\end{array}$ & $\begin{array}{l}0,001 \\
0,000 \\
0,000 \\
0,994\end{array}$ & $\begin{array}{l}0,012 \\
0,017 \\
0,000\end{array}$ \\
\hline $\begin{array}{l}\text { Estado civil (ref: casado o en pareja) } \\
\text { Soltero } \\
\text { Viudo, separado o divorciado }\end{array}$ & $\begin{array}{l}0,036 \\
0,114 \\
0,013 \\
\end{array}$ & $\begin{array}{l}0,159 \\
0,074\end{array}$ \\
\hline Constante & 0,194 & 3,927 \\
\hline
\end{tabular}


factores asociados a la recepción de cuidado informal, la tabla 4 los asociados a cuidado formal, y la tabla 5 los relacionados con la recepción de cuidado formal e informal conjuntamente.

El Modelo 1 (tabla 3) compara las características de (a) aquéllos que no recibían cuidado y (b) quienes sólo recibían cuidado informal. Los odds ratio indican que las mujeres tenían la mitad de probabilidades que los varones de recibir cuidado informal. A medida que aumentaba el tamaño del hogar se incrementaba significativamente la probabilidad de recibir apoyo informal. Los viudos, separados o divorciados tenían tres veces más probabilidades de recibir cuidado informal que los casados. En relación con aquéllos que sólo experimentaban problemas físicos, los que padecían problemas mentales o mentales y físicos tenía 3,9 veces más probabilidades de recibir cuidado informal. El modelo seleccionaba como significativa la variable tipo de problema, cuando habría cabido esperar que apareciera el nivel de dificultad. Ambas variables ofrecen información similar, dado que el $86 \%$ de las personas que padecían problemas mentales experi- mentaban también problemas físicos. Estos resultados sugieren que, de cara a la recepción de cuidado informal, el padecimiento de problemas mentales y físicos combinados es más determinante que el grado de dificultad en las AVD. La $\mathrm{R}^{2}$ de Nagelkerke es 0,173 , lo que refleja un aceptable ajuste del modelo. En muchas ocasiones, tanto las personas mayores dependientes como sus familias se planteaban dos opciones de cara al cuidado: (a) que el cuidado fuera provisto por los familiares o amigos, o (b) que se contratara a alguien para que realizara ese trabajo. De acuerdo con el Modelo 2 (tabla 3) la probabilidad de recibir cuidado de empleados de hogar en lugar de cuidado informal estaba relacionada con el tamaño de hogar, el nivel educativo y los ingresos. En comparación con los mayores sin estudios, los mayores con estudios secundarios o superiores tenían 4 veces más probabilidades de recibir cuidado de empleados de hogar que de familiares o amigos. Los mayores que vivían en hogares con más de $600 €$ mensuales tenían entre 3 y 5 veces más probabilidades de recibir cuidado de un cuidador pagado en lugar de cuidado informal que los hogares con menos ingre-

Tabla 4

Modelos de regresión logística de los factores asociados al cuidado formal (privado o público)

\begin{tabular}{|c|c|c|}
\hline \multicolumn{3}{|c|}{ Modelo 4. Recibe cuidado de un cuidador remunerado (ref: no recibe ningún tipo de cuidado) } \\
\hline Variables & Sig. & Odds ratio \\
\hline Edad (ref: $65-74$ ) & 0,011 & \\
\hline $75-84$ & 0,061 & 2,075 \\
\hline Más de 84 & 0,003 & 4,579 \\
\hline Constante & 0,005 & 0,429 \\
\hline \multicolumn{3}{|c|}{ Porcentaje pronosticado correctamente por el modelo: $61,445 \% . \mathrm{R}^{2}$ de Nagelkerke: 0,087 . Modelo por pasos hacia delante. Paso $1^{\circ}$. Edad. } \\
\hline \multicolumn{3}{|c|}{ Modelo 5. Recibe cuidado de servicios sociales (ref: no recibe ningún tipo de cuidado) } \\
\hline Variables & Sig. & Odds ratio \\
\hline $\begin{array}{l}\text { Edad (ref: } 65-74) \\
75-84 \\
\text { Más de } 84\end{array}$ & $\begin{array}{l}0,017 \\
0,158 \\
0,004\end{array}$ & $\begin{array}{l}1,975 \\
6,285\end{array}$ \\
\hline $\begin{array}{l}\text { Ingresos del hogar (ref: menos de } 600 €) \\
601-900 € \\
\text { Más de } 900 € \\
\text { No consta }\end{array}$ & $\begin{array}{l}0,010 \\
0,739 \\
0,021 \\
0,015 \\
\end{array}$ & $\begin{array}{l}1,182 \\
0,107 \\
0,147\end{array}$ \\
\hline Constante & 0,019 & 0,392 \\
\hline
\end{tabular}


Tabla 5

Modelos de regresión logística de los factores asociados al cuidado formal e informal conjuntamente

\begin{tabular}{|c|c|c|}
\hline \multicolumn{3}{|c|}{ Modelo 6. Recibe cuidado privado y cuidado informal (ref: recibe sólo un tipo de cuidado) } \\
\hline Variables & Sig. & Odds ratio \\
\hline $\begin{array}{l}\text { Sexo (ref: Varón) } \\
\text { Mujer }\end{array}$ & 0,001 & 3,489 \\
\hline $\begin{array}{l}\text { Edad (ref: 65-74) } \\
75-84 \\
\text { Más de } 84\end{array}$ & $\begin{array}{l}0,026 \\
0,111 \\
0,007\end{array}$ & $\begin{array}{l}1,798 \\
2,867\end{array}$ \\
\hline $\begin{array}{l}\text { Nivel de dificultad (ref: moderada) } \\
\text { Severa }\end{array}$ & 0,000 & 2,639 \\
\hline $\begin{array}{l}\text { Tamaño del hogar (ref: } 1) \\
2 \\
3 \\
4 \text { o más }\end{array}$ & $\begin{array}{l}0,031 \\
0,078 \\
0,003 \\
0,112\end{array}$ & $\begin{array}{l}0,439 \\
0,170 \\
0,447\end{array}$ \\
\hline $\begin{array}{l}\text { Estado civil (ref: casado o en pareja) } \\
\text { Soltero } \\
\text { Viudo, separado o divorciado }\end{array}$ & $\begin{array}{l}0,051 \\
0,048 \\
0,888\end{array}$ & $\begin{array}{l}2,466 \\
0,950\end{array}$ \\
\hline $\begin{array}{l}\text { Nivel educativo (ref: sin estudios) } \\
\text { Estudios primarios o equivalentes } \\
\text { Estudios secundarios o superiores }\end{array}$ & $\begin{array}{l}0,000 \\
0,000 \\
0,001\end{array}$ & $\begin{array}{l}3,536 \\
4,186\end{array}$ \\
\hline $\begin{array}{l}\text { Ingresos del hogar (ref: menos de } 600 €) \\
601-900 € \\
\text { Más de } 900 € \\
\text { No constan ingresos }\end{array}$ & $\begin{array}{l}0,031 \\
0,994 \\
0,117 \\
0,012\end{array}$ & $\begin{array}{l}0,996 \\
2,088 \\
3,157\end{array}$ \\
\hline $\begin{array}{l}\text { Tamaño de municipio (ref: menos de } 10.000 \text { ) } \\
10.001-100.000 \\
\text { Más de } 100.000\end{array}$ & $\begin{array}{l}0,012 \\
0,003 \\
0,036\end{array}$ & $\begin{array}{l}3,297 \\
2,339\end{array}$ \\
\hline Constante & 0,000 & 0,003 \\
\hline \multicolumn{3}{|c|}{$\begin{array}{l}\text { N: 903. Porcentaje pronosticado correctamente por el modelo: } 90,949 \% \text {. } \mathrm{R}^{2} \text { de Nagelkerke: } 0,236 \text {. Modelo por pasos hacia delante. Paso } 1^{\circ} \text {. Nivel de } \\
\text { estudios. Paso } 2^{\circ} \text {. Estado civil. Paso } 3^{\circ} \text {. Nivel de dificultad. Paso } 4^{\circ} \text {. Sexo. Paso } 5^{\circ} \text {. Tamaño del municipio. Paso } 6^{\circ} \text {. Edad. Paso } 7^{\circ} \text {. Ingresos del hogar } \\
\text { Paso } 8^{\circ} \text {. Tamaño del hogar }\end{array}$} \\
\hline \multicolumn{3}{|c|}{ Modelo 7. Recibe cuidado de servicios sociales y cuidado informal (ref: recibe sólo un tipo de cuidado) } \\
\hline Variables & Sig. & Odds ratio \\
\hline $\begin{array}{l}\text { Edad (ref: } 65-74) \\
75-84 \\
\text { Más de } 84\end{array}$ & $\begin{array}{l}0,009 \\
0,005 \\
0,003\end{array}$ & $\begin{array}{l}5,130 \\
6,294\end{array}$ \\
\hline $\begin{array}{l}\text { Nivel de dificultad (ref: moderada) } \\
\text { Severa }\end{array}$ & $\begin{array}{l}0,000 \\
0,000\end{array}$ & 4,111 \\
\hline Tamaño del hogar (ref: 1) & $\begin{array}{l}0,228 \\
0,676 \\
0,050 \\
0,994\end{array}$ & $\begin{array}{l}0,837 \\
0,323 \\
0,000\end{array}$ \\
\hline Constante & 0,000 & 0,010 \\
\hline
\end{tabular}

sos. La $\mathrm{R}^{2}$ de Nagelkerke reflejó un buen ajuste del modelo (0,251). El Modelo 3 (tabla 3) comparaba (a) quienes recibían cuidado informal con (b) quienes recibían cuidado de servicios sociales. Quienes vivían solos tenían más probabilidades de recibir cuidado de servicios sociales en lugar de cuidado informal. Los casados o en pareja tenían significativamente más probabilidades que los viudos, separados o divorciados (esta categoría se compone de
585 viudos, 5 separados legalmente y 4 divorciados) de recibir atención de servicios sociales. La $\mathrm{R}^{2}$ de Nagelkerke $(0,273)$ indica un elevado ajuste del modelo.

De acuerdo con el Modelo 4 (tabla 4), aquellas personas mayores que superaban los 84 años tenía 4 veces y media más probabilidades de recibir cuidado privado en lugar de ningún tipo de cuidado que quienes tenían entre 65 y 74 años. Llamó la aten- 
ción la ausencia en el modelo de la variable ingresos. Asimismo, fue notorio que no apareciera como significativo el tipo de problema (o el nivel de dificultad), cuando sí está presente en la comparación entre el cuidado informal y la no asistencia. Una posible explicación a estas ausencias es el limitado número de casos del modelo (206). La $\mathrm{R}^{2}$ de Nagelkerke es 0,087 lo que indica que el ajuste del modelo no es elevado. El Modelo 5 (tabla 4) refleja que el cuidado por parte de servicios sociales era más de 6 veces más probable entre quienes superan los 84 años que en la cohorte 65-74 años. Quienes vivían en hogares de menos de 600 $€$ mensuales tenían cerca de diez veces más probabilidades de recibir servicios públicos que quienes vivían en hogares que superan $\operatorname{los} 900 €$.

El Modelo 6 (tabla 5) explora la probabilidad de recibir cuidado informal y cuidado privado conjuntamente, en comparación con la recepción de un solo tipo de cuidado (en el $82 \%$ de los casos cuidado informal). La probabilidad de recibir cuidado informal y privado combinado fue mayor para las mujeres (tres veces y media más probabilidades), los mayores de 84 años (casi tres veces más), quienes tenían dificultades severas (2,6 veces más), vivían solos (en relación con quienes viven en hogares de tres personas), estaban solteros (2,5 veces más probabilidades que los casados), tenían niveles de estudios más elevados (hasta cuatro veces más) y vivían en hogares de municipios que superaban los 10.000 habitantes. La $\mathrm{R}^{2}$ de Nagelkerke es 0,236, lo que indicaba un buen ajuste del modelo. La recepción de cuidado informal y de servicios sociales conjuntamente (tabla 5, Modelo 7) se encontraba con más probabilidad en las edades más avanzadas (hasta seis veces más), niveles de dificultad severa (cuatro veces más) y hogares unipersonales. Aunque cabría esperar lo contrario, los ingresos del hogar no aparecían como factor significativo. El ajuste del modelo fue aceptable $\left(\mathrm{R}^{2}\right.$ de Nagelkerke 0,200).

\section{DISCUSIÓN}

Este trabajo aporta nueva información sobre la distribución del cuidado formal e informal en España y sobre el modo en que se relacionan. Se observa una presencia mayoritaria del modelo de cuidado complementario en combinación con el modelo compensador jerárquico: el cuidado formal es minoritario y sirve de complemento al informal, que es el tipo de cuidado prioritario y mayoritario. Las personas mayores con necesidades de atención que viven acompañados (o su entorno) parecen escoger como primera opción el cuidado informal, de acuerdo con el modelo compensador jerárquico. En la línea de lo hallado por Bolin et al. ${ }^{26}$ para las personas mayores en diez países europeos, entre ellos España, se confirma que la relación entre cuidado formal e informal entre quienes viven solos es de sustitución (modelo de cuidado suplementario). Se constata a su vez que los servicios públicos de cuidado tienen una menor presencia que los servicios privados en España ${ }^{27}$. No es frecuente que se combine cuidado formal e informal, aunque las probabilidades de que esto ocurra aumentan cuando el nivel de dificultad es mayor. Estos resultados se sitúan en consonancia con el trabajo de Jiménez-Martín y Vilaplana para España con datos de $1999^{28}$, que hallaron una mayor presencia del modelo complementario y que el modelo suplementario era minoritario. Los resultados también reflejan que hay un porcentaje relevante de mayores que declaran necesitar ayuda y no la reciben.

Debido a la escasez de investigaciones realizadas en España sobre los factores que influyen en la recepción de cuidado formal e informal, es conveniente contrastar los resultados de este trabajo con estudios realizados en otros países, aunque no se trate de investigaciones recientes. Este trabajo confirma que un mayor porcentaje de mujeres no recibe cuidado a pesar de necesitarlo, que los hombres reciben más cuidado infor- 
mal como única fuente de apoyo, y que las mujeres reciben más cuidado formal, tal y como sugiere el estudio de Pérez Ortiz para España con información de la Encuesta de Condiciones de Vida de los Mayores $2004^{29}$, y el de Katz el al. para Estados Unidos con datos de $1993^{30}$.

Como era de esperar, la presencia del tamaño del hogar en todos los modelos sobre cuidado informal confirma la relevancia de la red social más cercana en la recepción de este tipo de apoyo. El tamaño del hogar puede ser el resultado de un cambio de convivencia a causa de la dependencia. Con la fuente utilizada, no es posible saber si el cuidado informal es consecuencia del tamaño del hogar o si el número de miembros ha aumentado por la necesidad de proveer la atención en el hogar del cuidador informal. El cuidado formal público es más frecuente en los hogares con menos miembros, lo que no debe extrañar si se tiene en cuenta que uno de los criterios seleccionados por algunas administraciones para la provisión de servicios públicos es la ausencia de familiares en el entorno, tal y como señala el Libro Blanco de la Dependencia ${ }^{1}$.

La recepción de cuidado formal privado, como única fuente de apoyo, está relacionada con la disponibilidad de recursos socioeconómicos y no tanto con el grado de dificultad o el tipo de problema que genera la dependencia, en la línea de lo encontrado por Kemper en Estados Unidos (datos de 1988). Este hallazgo es consistente con los resultados de Phillips sobre una población de rentas bajas en Estados Unidos en 1991 y 1992, que no halló diferencias significativas en la recepción de cuidado formal según nivel de dependencia. En cambio, la probabilidad de recibir cuidado formal e informal combinado aumenta considerablemente entre quienes tienen dificultades más severas, lo que se sitúa en consonancia con el estudio de Chappell y Blandford para Canadá.
Los resultados indican que los casados tienen significativamente menos probabilidades de recibir cuidado informal que los viudos, separados o divorciados, y más probabilidades de recibir cuidado de servicios sociales en lugar de cuidado informal. Una explicación sugerida por Delicado ${ }^{31}$ es que los cónyuges actúan como conectores entre la persona mayor y el sistema público de salud y/o cuidados, especialmente cuando la carga es excesiva o se demandan habilidades que sólo pueden ser desarrolladas por profesionales. Por otra parte, podría existir un sesgo en la identificación del tipo de cuidado por parte de los casados: es posible que quienes reciben cuidado informal de su cónyuge no lo identifiquen como tal, sino como un tipo de ayuda normal, difícilmente distinguible de la que se proveía antes de la aparición de la dependencia. Las características del cuidado formal (burocratización, etc.) lo hacen más fácilmente identificable.

El nivel educativo aumenta la probabilidad de recibir cuidado formal y reduce la de cuidado informal, en consonancia con lo hallado por Otero et al. en una ciudad del área metropolitana de Madrid ${ }^{32}$. Una posible explicación es que el nivel educativo de la persona mayor está relacionado con el nivel educativo de sus familiares, de modo que refleja el coste de oportunidad del cuidado informal en las personas de su entorno: el cuidado informal de los familiares de dependientes con mayores niveles educativos tendrá un coste de oportunidad más elevado e intentarán, en la medida de lo posible, sustituirlo por cuidado formal, tal y como sugiere el citado estudio de JiménezMartín y Vilaplana ${ }^{28}$.

Los principales límites de este trabajo residen en la muestra utilizada. Ésta se refiere a personas no institucionalizadas, y tiene un tamaño que no permite profundizar en algunas de las relaciones encontradas. Se recomienda que la investigación futura sobre la relación entre cuidado for- 
mal e informal se apoye en fuentes de mayor tamaño y, a ser posible, de carácter longitudinal. En esta línea, es conveniente utilizar fuentes recientes, como la Encuesta Nacional de Salud 2006 (INE) y la Encuesta sobre Discapacidades, Autonomía Personal y Situaciones de Dependencia 2008 (INE), para contrastar los hallazgos de este trabajo y conocer la evolución del modelo de atención a las personas mayores en España.

\section{BIBLIOGRAFÍA}

1. IMSERSO. Libro Blanco de la Dependencia. Madrid: Instituto de Mayores y Servicios Sociales; 2005

2. Andersson A, Levin, L-A y Emtinger BG. The economic burden of informal care. International. Int J Technol Assess Health Care. 2002; 18(1): 46-54.

3. Pentland WE y McColl MA. Application of Time Use Research to the Study of Life with a Disability. En Pentland, WE, Harvey, AS, Lawton, MP, y McColl, MA, editores. "Time Use Research in the Social Sciences". New York: Kluwer Academic / Plenum Publishers; 1999. p. 169-188.

4. Rodríguez Rodríguez P. El apoyo informal y su complementariedad con los recursos formales. En Puyol, R y Abellán, A, editores. Envejecimiento y dependencia. Una mirada al panorama futuro de la población española. Madrid: Mondial Assistance; 2006. pp. 148-168.

5. Rogero-García J, Prieto-Flores ME y Rosenberg MW. Health services use by older people with disabilities in Spain: do formal and informal care matter? Ageing Soc. 2008; 28 (7): 959-978.

6. Francisco del Rey C y Mazarrasa Alvear L. Cuidados informales. Rev Enferm. 1995; 18(202): 6165 .

7. Durán MA. Los costes invisibles de la enfermedad. $2^{\mathrm{a}}$ ed. Bilbao: Fundación BBVA; 2002.

8. Chappell $\mathrm{N}$ y Blandford $\mathrm{A}$. Informal and formal care: exploring the complementarity. Ageing Soc. 1991; 11(3): 299-317.

9. Phillips V. Community care for severely disabled people on low incomes. BMJ. 1995; 311(7013): 1121-1123.
10. Kemper P. The use of formal and informal home care by the disabled elderly. Health Serv Res. 1992; 27(4): 421-451.

11. Herlitz C. Distribution of Informal and Formal Home Help for Elderly People in Sweden. Gerontologist. 1997; 37(1): 117-124.

12. Castejón P, Esparza C \& Abellán A. Salud, dependencia y cuidados. En IMSERSO, editor. A propósito de las condiciones de vida de las personas mayores. Madrid: IMSERSO; 2007. pp. 65-82.

13. Ungerson C. Policy is personal. Sex, gender, and informal care. London: Tavistock Publications; 1987.

14. Walker AJ, Pratt CC \& Eddy L. Informal Caregiving to Ageing Family Members: A critical review. Fam Relat. 1995; 44(4): 402-411.

15. Twigg J. Cuidadores de los ancianos: modelos para un análisis. En Jamieson A y Illsley R, editores. Comparación de políticas europeas de atención a las personas ancianas. Barcelona: SG Editores; 1993. pp. 35-51.

16. Pearlin LI y Zarit SH. Research into Informal Caregiving: Current Perspectives and Future Directions. En Zarit SH, Pearlin LI \& Schaie, KW, editores. Caregiving Systems. Formal and Informal Helpers. Hillsdale: Lawrence Erlbaum Associates; 1993. pp. 155-167.

17. Real Academia Española de la Lengua [citado 9 sep. 2008]. http://www.rae.es.

18. Davey A y Patsios D. Formal and Informal Community Care to Older Adults: Comparative Analysis of the United States and Great Britain. J Fam Econ Issues. 1999, 20(3).

19. Keating N, Fast J, Forbes D y Wenger C. Informal Care Networks of Canadian Seniors with LongTerm Health Problems. Edmonton: Research on Aging. Policies and Practice (RAPP). University of Alberta; 2003.

20. García Calvente MM \& Mateo Rodríguez I. El cuidado de la salud ¿quién hace qué? Index de Enfermería. 1993; 6: 16-19.

21. Exel JV, Morée M, Koopmanschap M, Goedheijt TS y Brouwer W. Respite care - An explorative study of demand and use in Dutch informal caregivers. Health Policy. 2006; 78: 194-208.

22. INE. Encuesta Nacional de Salud. Metodología. Instituto Nacional de Estadística; 2003. 
23. INE. Encuesta Nacional de Salud. Metodología. Instituto Nacional de Estadística; 2003.

24. Harrel FE. Regression Modeling Strategies. With Applications to Linear Models, Logistic Regression and Survival Analysis. New York: Springer; 2001.

25. Norusis M. SPSS 13.0. Advanced Statistical Procedures Companion. New Jersey: Prentice Hall; 2005.

26. Bolin K, Lindgren B y Lundborg P. Informal And Formal Care Among Single-Living Elderly In Europe. Tinbergen Institute Discussion Papers 2007; TI2007031/3. Amsterdam: Tinbergen Institute.

27. Bazo MT y Ancizu I. El papel de la familia y los servicios en el mantenimiento de la autonomía de las personas mayores: una perspectiva internacional comparada. Rev Esp Invest Sociol. 2004; 105: 43-77.
28. Jiménez-Martín S y Vilaplana Prieto C. Trade-off between formal and informal care in Spain. Documentos de Trabajo FEDEA. 2008, 22.

29. Pérez Ortiz L. La estructura social de la vejez en España. Nuevas y viejas formas de envejecer. Madrid: IMSERSO; 2006.

30. Katz S, Kabeto M y Langa K. Gender disparities in the receipt of home care for elderly people with disability in the United States. JAMA. 2000; 284(23): 3022-3027.

31. Delicado MV. Familia y cuidados de salud. Calidad de vida en cuidadores y repercusiones sociofamiliares de la dependencia [tesis doctoral]. Alicante: Universidad de Alicante; 2003.

32. Otero A, García de Yebenes MJ, Rodriguez-Laso A y Zunzunegui MV. Unmet home care needs among community-dwelling elderly people in Spain. Aging Clin Exp Res. 2003; 15(3): 234-242. 
